\title{
Nanoscale
}

A) Check for updates

Cite this: Nanoscale, 2020, 12, 12263

Received 8th April 2020,

Accepted 12th May 2020

DOI: $10.1039 / d 0 n r 01919 d$

rsc.li/nanoscale

\section{Scalable growth of single-walled carbon nanotubes with a highly uniform structure $\uparrow$}

\author{
Aqeel Hussain, (DD a Er-Xiong Ding, (DD a Ben Mclean, (D) ${ }^{\mathrm{b}}$ Kimmo Mustonen, ${ }^{\mathrm{c}}$ \\ Saeed Ahmad, ${ }^{a}$ Mohammad Tavakkoli, (D) a Alister J. Page, (D) ${ }^{b}$ Qiang Zhang, ${ }^{\text {a }}$ \\ Jani Kotakoski iD c and Esko I. Kauppinen*a
}

\begin{abstract}
Here, a scalable floating catalyst chemical vapor deposition (FCCVD) method is developed for the production of single-walled carbon nanotubes (SWCNTs) with a controlled structure. For the first time, water is used as the growth promoter in the FCCVD process to modulate the growth of SWCNTs. At an optimum water concentration of $\mathrm{ca} .115 \mathrm{ppm}$, the water-assisted FCCVD process synthesizes SWCNTs with a significantly narrow chirality distribution. In particular, the proportion of $(9,8)$ and $(8,7)$ semiconducting tubes was dramatically enhanced to $45 \%$ with $27 \%$ of the $(9,8)$ tube in the end product. This is attributed to the changes in both the SWCNT diameter and the chiral angle. The experiment results and accurate quantum chemical molecular dynamics simulations show that the addition of water affects the nucleation and the size distribution of nanoparticle catalysts, thus resulting in the growth of SWCNTs with a highly uniform structure. This direct and continuous water-assisted FCCVD provides the possibility for the mass production of high-quality SWCNTs with a controlled structure.
\end{abstract}

Control over the diameter and chirality of single-walled carbon nanotubes (SWCNTs) is one of the major obstacles to their application. ${ }^{1}$ The electronic properties of SWCNTs are so sensitive to their structure that even a slight shift in nanotube chirality results in significant changes in their electronic band structure. ${ }^{2}$ For example, chirality determines the metallic or semiconducting nature of the tubes. ${ }^{3,4}$ SWCNTs are produced with different structures and this structural heterogeneity limits their applications. SWCNTs with narrow chirality distribution have narrow bandgap which reduces the device performance variations, e.g., in the on/off ratio of a transistor. Uniform devices are the main target for applications, this is

\footnotetext{
${ }^{a}$ Department of Applied Physics, Aalto University School of Science, P.O. Box 15100, FI-00076 Aalto, Finland.E-mail: qiang.zhang@aalto.fi, esko.kauppinen@aalto.fi ${ }^{b}$ School of Environmental \& Life Sciences, University of Newcastle, Callaghan 2308, Australia

${ }^{c}$ Faculty of Physics, University of Vienna, Boltzmanngasse 5, 1090 Vienna, Austria $\dagger$ Electronic supplementary information (ESI) available. See DOI: 10.1039/ d0nr01919d
}

why single or the narrowest possible chirality distribution tubes are needed for applications in field-effect transistors (FETs) ${ }^{5}$ integrated circuits $^{6}$ and other nanoelectronics. Moreover, for semiconducting tubes, the energy band structure of a SWCNT is inversely proportional to the diameter. ${ }^{7}$ Due to their large band gaps, small diameter SWCNTs provide a very high on/off ratio in transistor applications, and a fast spectral response in optoelectronics. ${ }^{8}$

Tremendous efforts have been devoted to the growth of SWCNTs with a controlled chirality. There have been attempts to directly grow SWCNTs with a defined chirality. An important method for this is the selective synthesis of SWCNTs using well-controlled and high-temperature stabilized catalyst nanoparticles (NPs) on a substrate. ${ }^{9,10}$ Selective etching is another well-known strategy for the controlled growth of SWCNTs in an oxidative environment of oxygen, ${ }^{11}$ water, ${ }^{12}$ ammonia ${ }^{13}$ and carbon dioxide. ${ }^{14,15}$ Despite the progress, there are significant obstacles to the mass production of SWCNTs with controlled structures $^{9,16,17}$ for practical applications. Substrate-CVD produces SWCNTs at a low yield and has limited potential for upscale production. However, floating catalyst chemical vapor deposition (FCCVD) has demonstrated potential for the direct and continuous manufacturing of SWCNTs at a high yield, while maintaining their intrinsic electronic properties and eliminating the need for time-consuming solution processing. ${ }^{18}$

We have developed water-assisted floating catalyst chemical vapor deposition (FCCVD) for the scalable synthesis of SWCNTs with obviously narrow chirality distribution. FCCVD has already been scaled up for large-scale synthesis of SWCNTs. This is the first time that $\mathrm{H}_{2} \mathrm{O}$ has been used as a promoter for structure-controlled SWCNT synthesis by FCCVD. Water has only been used in substrate-CVD before for the growth of SWCNTs. ${ }^{19-21} \mathrm{H}_{2} \mathrm{O}$ addition increases the yield of SWCNTs over 100-fold. We were able to mass synthesize SWCNTs with a high enrichment $(45 \%)$ of two dominant chiral indices $(9,8)$ and $(8,7)$ tuned from near-random chirality distribution. In comparison, our work produces SWCNTs with 
narrower chirality distribution than several other studies using FCCVD. ${ }^{13,14,22}$ Scaling up the production of SWCNTs with highly controlled chirality will play an essential role for their commercial applications.

We studied the effect of water on highly individual SWCNTs grown using 0.75 standard cubic centimeter per minute (sccm) ethylene, $45 \mathrm{ccm}$ hydrogen and $40 \mathrm{sccm}$ of $\mathrm{N}_{2}$ through the ferrocene cartridge (Fig. $\mathrm{S} 1 \dagger){ }^{22}$ The yield was calculated by the transmittance of SWCNT films based on the Beer-Lambert law. $^{23}$ The reaction yield is significantly increased upon adding water (Fig. 1a). For 115 ppm $\mathrm{H}_{2} \mathrm{O}$, the reaction yield increases more than 10 times compared to 0 ppm water and reaches a maximum value at $300 \mathrm{ppm} \mathrm{H}_{2} \mathrm{O}$ with a more than 100 -fold increase in the yield. However, once the $\mathrm{H}_{2} \mathrm{O}$ concentration exceeds $300 \mathrm{ppm}$ the yield decreases. To further clarify the increase of the yield, a Differential Mobility Analyzer (DMA) was employed for the online analysis. ${ }^{24}$ DMA profiles indicate the increase of both particle and nanotube concentrations upon adding water (Fig. S2b $\dagger$ ). The concentration of SWCNTs is negligible at $0 \mathrm{ppm}$ water, which increases to $1.2 \times 10^{6}$ nanotubes per $\mathrm{cm}^{3}$ for the addition of $115 \mathrm{ppm}$ water. DMA analysis also showed a maximum yield at $300 \mathrm{ppm}$ with concentration $2 \times 10^{6}$ nanotubes per $\mathrm{cm}^{3}$. It confirms the increase in the nanotube synthesis yield for the addition of water.

Optical absorption spectroscopy allows rapid quantification of the SWCNT diameter. The absorption spectra of the SWCNT films are shown in Fig. 1b. With an increase in the $\mathrm{H}_{2} \mathrm{O}$ concentration, the transition peak $\mathrm{S}_{11}$ from the semiconducting tubes first has a blue-shift at lower water concentrations ( 0 to $140 \mathrm{ppm}$ ) and then red-shift at high water concentrations (190 to $350 \mathrm{ppm})$. From the Kataura plot and the absorption spectra, the mean diameter of SWCNTs was calculated. ${ }^{25,26}$ The mean diameter of SWCNTs is $1.30 \mathrm{~nm}$ in the absence of $\mathrm{H}_{2} \mathrm{O}$, which significantly decreases to $1.05 \mathrm{~nm}$ with the addition of a small amount of $\mathrm{H}_{2} \mathrm{O}$ (50 to $140 \mathrm{ppm}$ ). However, the SWCNT diameter unexpectedly increases with more water addition (190 to $350 \mathrm{ppm}$ ) resulting in a mean diameter of $1.20 \mathrm{~nm}$ at $300 \mathrm{ppm} \mathrm{H}_{2} \mathrm{O}$. The absorption peaks $\left(S_{11}\right.$ and $\left.S_{22}\right)$ of SWCNTs synthesized at a small concentration of water (50 to $140 \mathrm{ppm}$ ) are obviously narrower compared to the peaks a)

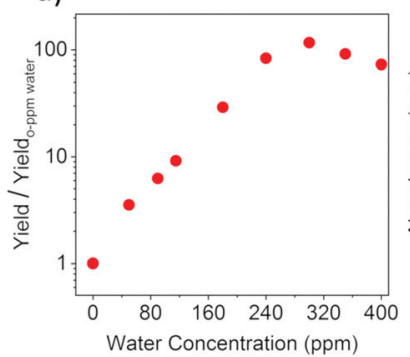

b)

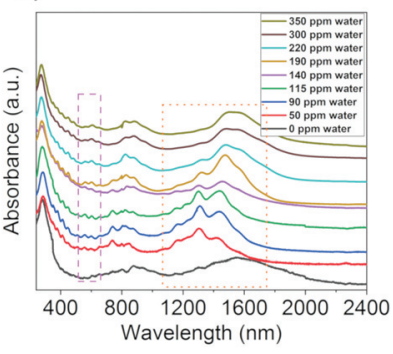

Fig. 1 (a) Normalized SWCNT synthesis yield at varied $\mathrm{H}_{2} \mathrm{O}$ concentrations. (b) Absorption spectroscopy indicates variation in nanotube diameters at different $\mathrm{H}_{2} \mathrm{O}$ concentrations. arising from the nanotubes produced in the absence of water. This suggests that water addition produces SWCNTs with a narrower diameter distribution. The SWCNT diameter variations due to the addition of water are supported by the analysis from Raman spectra (Fig. S4 $\dagger$ ). Raman spectroscopy also showed the growth of small diameter tubes at $115 \mathrm{ppm}_{2} \mathrm{O}$.

Electron diffraction analysis was used to study the chiral structure of individual SWCNTs. The SWCNTs were deposited on the TEM grid and each tube was approached separately to assess its diffraction pattern. The diffraction patterns of different types of tubes are shown in Fig. S5. $\dagger$ Based on the intrinsic layer line analysis method from the electron diffraction pattern, the chiral indices $(n, m)$ were assigned to individual tubes. ${ }^{27}$ We investigated SWCNTs grown at 0 ppm, $115 \mathrm{ppm}$ and at a high $\mathrm{H}_{2} \mathrm{O}$ concentration of $300 \mathrm{ppm}$, and their corresponding absorption spectra are shown in Fig. S3. $\dagger$ The statistical $(n, m)$ and diameter distribution of 120 individual SWCNTs for each condition are shown in Fig. 2 and S6. $\dagger$

Accurate chirality maps (Fig. 2) show that the addition of water affects diameter, chirality and chiral angle distribution of SWCNTs. As shown in Fig. 2d, the mean diameter of SWCNTs first decreases from $1.25 \mathrm{~nm}$ to $1.10 \mathrm{~nm}$ and then increases to $1.20 \mathrm{~nm}$ with increasing water concentration, which is consistent with the absorption results. The chiral angle distributions of SWCNTs are shown in Fig. S6b. $\dagger$ Without $\mathrm{H}_{2} \mathrm{O}$, the chiral angles are evenly and randomly dis-
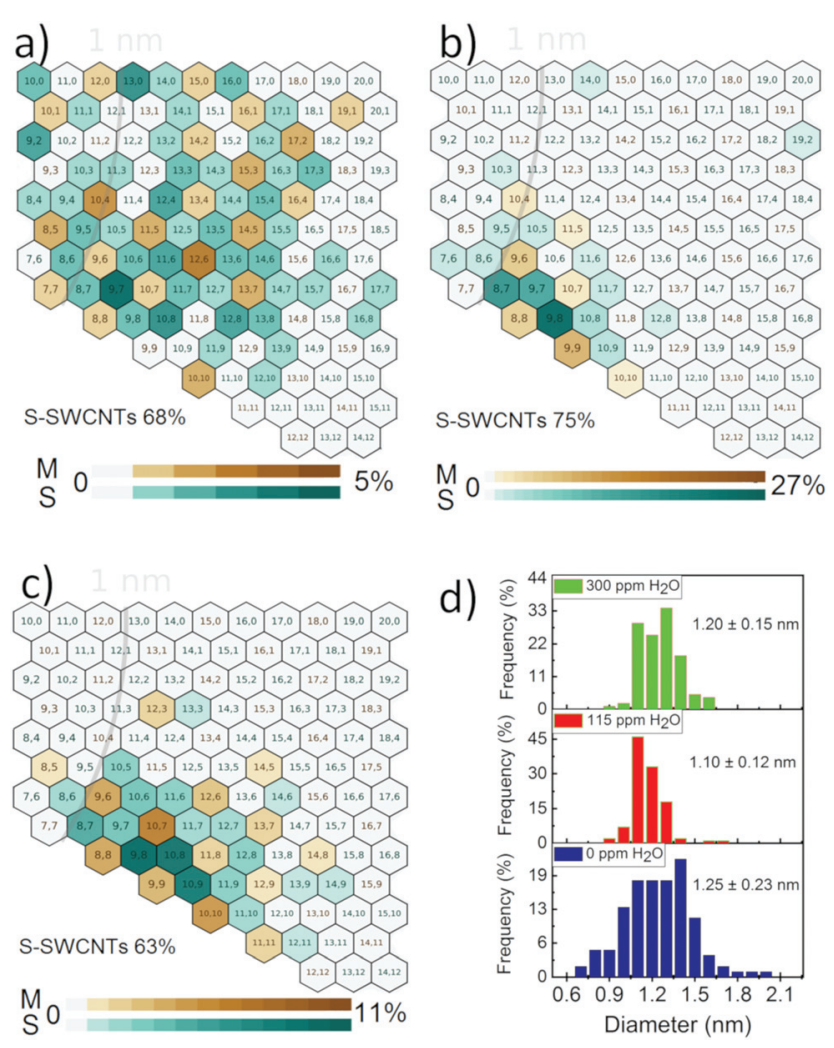

Fig. 2 Chirality distribution of 120 individual SWCNTs for the $\mathrm{H}_{2} \mathrm{O}$ concentration of (a) 0 ppm, (b) 115 ppm and (c) 300 ppm. (d) The nanotube diameter distribution obtained from electron diffraction measurements. 
tributed between 0 and $30^{\circ}$ with a mean chiral angle of $16^{\circ}$. However, SWCNTs are accumulated near the armchair edge at high chiral angles with the addition of water, which is in accordance with the screw dislocation theory. ${ }^{28}$ The optimized 115 ppm $\mathrm{H}_{2} \mathrm{O}$, in particular, produced SWCNTs with an extremely narrow chirality distribution. More than $95 \%$ of the tubes produced at this concentration have chiral angles between $20^{\circ}$ and $30^{\circ}$ and $90 \%$ of the tubes have diameters between 1.05 and $1.30 \mathrm{~nm}$. Two dominant chiralities of $(9,8)$ and $(8,7)$ are semiconductive and their enrichment reaches to $45 \%$ of the tubes among which single chirality $(9,8)$ accounts for $27 \%$ of the SWCNTs in the sample. Evaluated by the standard deviations of chiral angle and diameter as well as the maximum enrichment of single chirality, SWCNTs produced with 115 ppm $\mathrm{H}_{2} \mathrm{O}$ have the narrowest chirality distribution reported in the FCCVD process (Table $\mathrm{S} 1 \dagger$ ). In addition, the semiconducting ratio at $115 \mathrm{ppm}_{2} \mathrm{O}$ increases to $75 \%$ from $68 \%$ at 0 ppm $\mathrm{H}_{2} \mathrm{O}$. Notably, the yield of these SWCNTs with controlled structures is an order of magnitude higher than that without water and from other substrate $\mathrm{CVD}^{9,10}$ methods which suffer from low yields due to their activity-limited catalyst. The yield further increases at higher water concentrations, but chirality scatters again (Fig. 2c). For example, the enrichment of $(9,8)$ decreases to $10 \%$ at $300 \mathrm{ppm}_{2} \mathrm{O}$.

Our results, revealing for the first time the impact of water addition in FCCVD on SWCNT synthesis, differ from the previous studies on water-assisted substrate CVD synthesis of SWCNTs. ${ }^{11,29}$ Water is believed to create an oxidative environment where low chiral angle and small diameter nanotubes are preferably etched due to their high reactivity. ${ }^{30,11,29}$ So, we propose that the oxidative environment of water selectively removed the low chiral angle tubes (Fig. S6b $\dagger$ ). Kimura et al. recently calculated the reaction energy of an $\mathrm{OH}$ radical $\left(\Delta E_{\mathrm{OH}}\right)$ for different chiralities and proposed that there is a selective removal of low chiral angle tubes in the presence of $\mathrm{OH}$ due to a high $\Delta E_{\mathrm{OH}} \cdot{ }^{31}$ This prevents the growth of nanotubes in a wide distribution of chiral angles and makes the distribution narrow at high chiral angles. Similarly, the addition of water selectively removed the small diameter (less than $1 \mathrm{~nm}$ ) SWCNTs as shown in diameter distribution (Fig. 2d). Despite this, the mean diameter of the SWCNTs is decreased at $115 \mathrm{ppm}$ water which suggests further investigations of the process are needed.

Previous studies have correlated variations in the diameter and structure of the SWCNTs to the NP catalyst size. ${ }^{20,21,32}$ To observe the effect of water on NP nucleation and the subsequent SWCNT growth, we studied ferrocene decomposition decoupled from SWCNT growth. We stopped the ethylene flow to prevent the carbon source entering the reactor and used DMA for online measurement of the effect of water on the decomposition of ferrocene. In the absence of water, the concentration of NPs is extremely low in the reaction zone and is below the DMA measurement limits (Fig. S2a $\dagger$ ). However, the addition of 115 ppm $\mathrm{H}_{2} \mathrm{O}$ enormously increases the NP number concentration and it approaches $1.4 \times 10^{7}$ NPs per $\mathrm{cm}^{3}$. The concentration of NPs increases for a higher concen- a)

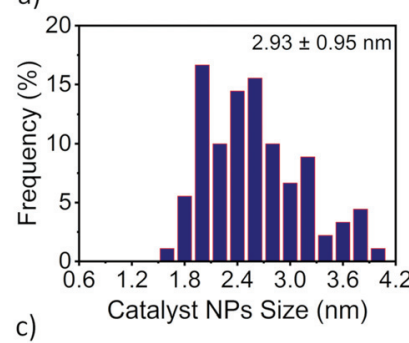

b)

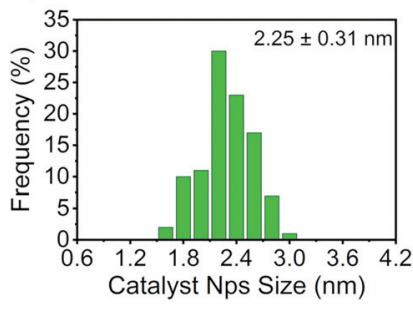

d)
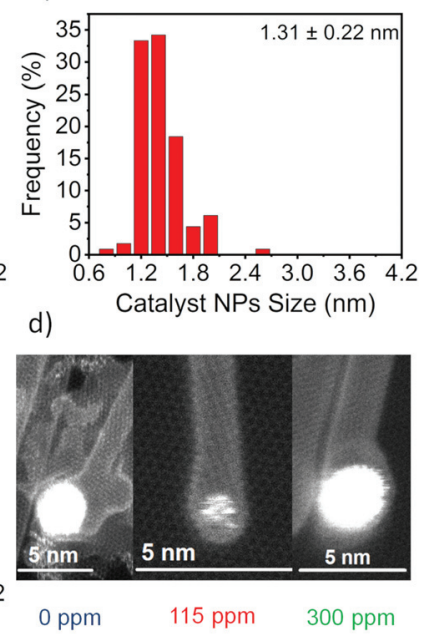

Fig. 3 Catalyst NP size distribution at (a) 0 ppm, (b) $115 \mathrm{ppm}$ and (c) $300 \mathrm{ppm}$. (d) High resolution STEM images of NPs at 0 ppm, $115 \mathrm{ppm}$ and $300 \mathrm{ppm} \mathrm{H}_{2} \mathrm{O}$.

tration of $\mathrm{H}_{2} \mathrm{O}$ and for DMA calculated to be $2.2 \times 10^{7}$ NPs per $\mathrm{cm}^{3}$ for $300 \mathrm{ppm}_{2} \mathrm{O}$. Water is proposed to change the NP formation mechanisms, i.e., the nucleation rate affecting the ferrocene homogeneous decomposition rate.

To further investigate the effect of water on the size of the NPs during the SWCNT growth, we used a scanning transmission electron microscope (STEM). The detailed STEM images of NPs for the growth of SWCNTs are shown in Fig. S7† and $3 \mathrm{~d}$. The NP size obviously decreases with the addition of a small amount of water. Fig. 3 shows a mean NP size of $2.93 \mathrm{~nm}$ at $0 \mathrm{ppm}$ water, which decreases to $1.31 \mathrm{~nm}$ with the addition of $115 \mathrm{ppm}$ of water. Moreover, the addition of $115 \mathrm{ppm}$ of water narrows down the NP size distribution. It shows that water impedes the size of NPs during nucleation. However, the highest water concentration of 300 ppm produced larger size NPs with a mean size of $2.25 \mathrm{~nm}$.

To explore the mechanism of the influence of water on catalyst NP nucleation, we performed quantum chemical molecular dynamics (MD) simulations of NP nucleation with and without an ambient water atmosphere. We observed that the nucleation of Fe NPs is influenced by the concentration of ambient water. Fig. 4a shows that the addition of water decreases the number of $\mathrm{Fe}-\mathrm{Fe}$ bonds formed during $\mathrm{Fe} \mathrm{NP}$ formation. We can relate this to the diameter of the NPs. Fig. $4 \mathrm{~b}$ shows the Fe NP diameter as a function of the water concentration. When no water is present, there is no impediment to Fe growth. Presumably the size of the catalyst particle here, $\sim 1.1 \mathrm{~nm}$, is only limited by the number of atoms present in the simulation. The addition of water to the simulation limits this diameter, via the dissociation of water molecules and formation of $\mathrm{Fe}-\mathrm{O}$ bonds on the NP surface. These $\mathrm{Fe}-\mathrm{O}$ bonds hinder further catalyst growth, and ultimately limits the particle size. For a given ferrocene density, this increases the concentration of NPs present, consistent with DMA and STEM measurements. At higher water concentrations, however, a 
a)

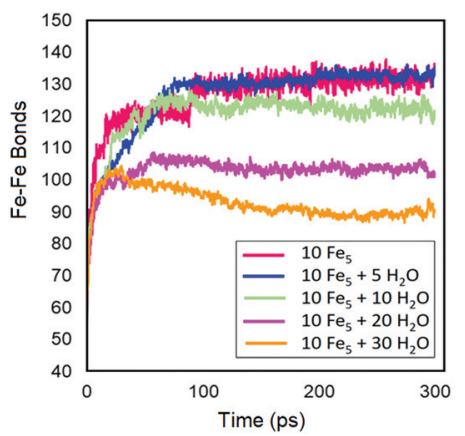

b)

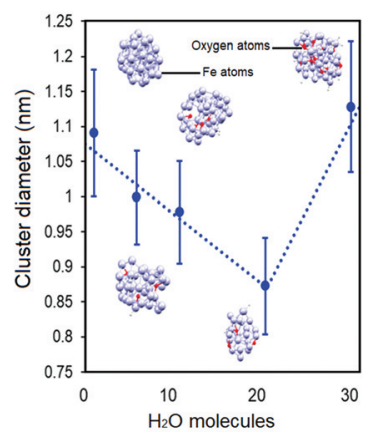

Fig. 4 (a) Rate of Fe-Fe bonding and (b) catalyst NP size distribution at 300 ps during DFTB simulations of 50 -atom catalyst NPs with a varying $\mathrm{H}_{2} \mathrm{O}$ molecule content at $1050{ }^{\circ} \mathrm{C}$.

shift to a larger NP size is observed (Fig. 4b). The incorporation of $\mathrm{O}$ into the NP structure is proposed to lead to an increase in the NP diameter at high water concentrations, despite the decreased number of Fe-Fe bonds shown in Fig. 4a. These observations are consistent with the larger NPs observed by STEM when the water concentration used during FCCVD is $300 \mathrm{ppm}$.

Our investigation suggests that the addition of water influences the catalyst formation, which ultimately affects the growth of SWCNTs. The small NPs, synthesized at $115 \mathrm{ppm}$ water, do not grow large diameter nanotubes, which is indicated by the absence of larger diameter (more than $1.3 \mathrm{~nm}$ ) tubes and a decrease in the mean diameter from $1.20 \mathrm{~nm}$ to $1.10 \mathrm{~nm}$, as shown in Fig. 2d. This, along with the removal of small diameter tubes (less than $1 \mathrm{~nm}$ ) due to an oxidative environment, compresses the diameter distribution of SWCNTs and makes it obviously narrow. In addition, the synthesis of more uniform size distributed NPs at 115 ppm water narrows down the diameter distribution of SWCNTs. So, the addition of water into the ethylene-based FCCVD produced SWCNTs with a highly uniform structure at a higher yield by narrowing the diameter and chiral angle distribution.

\section{Conclusions}

In summary, we achieved continuous synthesis of SWCNTs with a very narrow chirality distribution using water as a growth promoter. By optimizing the water concentration, the diameter of the nanotubes was reduced from $1.30 \mathrm{~nm}$ to $1.10 \mathrm{~nm}$. We observed an enormous shift in nanotube chirality from a random distribution at $0 \mathrm{ppm}$ to a narrow distribution at $115 \mathrm{ppm}$ with the content of chiral indices $(9,8)$ and $(8,7)$ enhanced to $45 \%$. The impact of water on the nucleation and growth of NPs accounts for the narrow diameter distribution. Etching of less stable zigzag SWCNTs and nucleation of narrow size distribution of catalyst NPs were proposed to elucidate the narrow chirality distribution of SWCNTs. Nanotubes with narrow chirality distribution are desirable for transistor devices and nanoelectronics.

\section{Experimental}

\section{Synthesis of SWCNTs}

SWCNTs were synthesized in an ethylene-based FCCVD reactor as described in our previous work (Fig. S1 $\dagger$ ). ${ }^{22}$ Briefly, 0.75 sccm of ethylene was used as the carbon source of which decomposition was balanced by $45 \mathrm{ccm}$ of $\mathrm{H}_{2}$. The flow of $\mathrm{N}_{2}$ through the ferrocene cartridge was fixed at $40 \mathrm{sccm} . \mathrm{H}_{2} \mathrm{O}$ vapor was introduced into the reactor by bubbling $\mathrm{N}_{2}$ gas through a water-filled bubbler kept at $21^{\circ} \mathrm{C}$. A flow of 0 to 40 sccm of $\mathrm{N}_{2}$ was passed through the water bubbler, corresponding to 0 to $350 \mathrm{ppm}$ of $\mathrm{H}_{2} \mathrm{O} .{ }^{12}$ The total flow through the reactor was maintained at $346 \mathrm{sccm}$ and the temperature of the reactor was fixed at $1050{ }^{\circ} \mathrm{C}$.

\section{Characterization}

The SWCNTs were characterized using various techniques including Raman spectroscopy (Horiba Jobin-Yvon Labram HR 800), optical absorption spectroscopy (Agilent Cary 5000) and electron diffraction (JEOL 2200FS Double Cs-corrected TEM). Chirality was determined by an intrinsic layer spacing method from the electron diffraction patterns. ${ }^{27}$ A Differential Mobility Analyzer (DMA, GRIMM Aerosol Technik GmbH \& Co. KG, Germany) was employed for the online tracking of the number, size and concentration of catalyst particles as well as SWCNTs. The measurement of catalyst size was achieved from scanning transmission electron microscopy (aberration-corrected Nion UltraSTEM 100) micrographs.

\section{Theoretical simulation}

The DFTB simulations employed the velocity-Verlet algorithm $^{33}$ to integrate Newton's equations of motion with a time step of 1.0 fs. An NVT ensemble was enforced at $1050{ }^{\circ} \mathrm{C}$ using a Nose-Hoover chain thermostat. ${ }^{34,35}$ The quantum chemical potential energy and energy gradients were calculated "on the fly" at each MD iteration using the SCC-DFTB method ${ }^{36}$ with the trans3d-0-1 parameter set $^{37}$ and a finite electronic temperature of $10000 \mathrm{~K}^{38-40}$

The influence of ambient water on the formation of Fe NPS during FCCVD was simulated via $10 \mathrm{Fe}_{5}$ clusters and either 0, $5,10,20$ or 30 water molecules in a cubic simulation box with a side length of $3 \mathrm{~nm}$. Trajectories were replicated 10 times for each simulation set (50 total trajectories), so that a representative chemical mechanism and statistically significant data were obtained.

\section{Conflicts of interest}

There are no conflicts to declare.

\section{Acknowledgements}

The research leading to these results has received partial funding from the European Union Seventh Framework 
Programme (FP7/2007-2013) under the Grant Agreement No. 604472 (IRENA project) and the Aalto Energy Efficiency (AEF) Research Program through the MOPPI project. The research has also been partially supported by the Academy of Finland via projects 286546 (DEMEC) and 292600 (SUPER) as well as by TEKES Finland via projects 3303/31/2015 (CNT-PV) and 1882/ 31/2016 (FEDOC). The authors acknowledge the Finnish Academy of Science and Letters for the financial support of this research work. This work made use of the Aalto University Nanomicroscopy Center (Aalto-NMC) premises.

\section{References}

1 Y. Chen, Y. Zhang, Y. Hu, L. Kang, S. Zhang, H. Xie, D. Liu, Q. Zhao, Q. Li and J. Zhang, Adv. Mater., 2014, 26, 58985922.

2 T. W. Odom, J.-L. Huang, P. Kim and C. M. Lieber, J. Phys. Chem. B, 1998, 391, 62-64.

3 T. W. Odom, J. L. Huang, P. Kim and C. M. Lieber, Nature, 1998, 391, 62-64.

4 A. Hassanien, M. Tokumoto, Y. Kumazawa, H. Kataura, Y. Maniwa, S. Suzuki and Y. Achiba, Appl. Phys. Lett., 1998, 73, 3839-3841.

5 A. D. Franklin, Science, 2015, 349, 6249.

6 D. Sun, M. Y. Timmermans, Y. Tian, A. G. Nasibulin, E. I. Kauppinen, S. Kishimoto, T. Mizutani and Y. Ohno, Nat. Nanotechnol., 2011, 6, 156-161.

7 R. Saito, G. Dresselhaus and M. S. Dresselhaus, Phys. Rev. B: Condens. Matter Mater. Phys., 2000, 61, 2981-2990.

8 D. j. Bindle, M. Wu, F. C. Prehn and M. S. Arnold, Nano Lett., 2011, 11, 455-460.

9 F. Yang, X. Wang, D. Zhang, J. Yang, D. Luo, Z. Xu, J. Wei, J. Q. Wang, Z. Xu, F. Peng, X. Li, R. Li, Y. Li, M. Li, X. Bai, F. Ding and Y. Li, Nature, 2014, 510, 522-524.

10 S. Zhang, L. Kang, X. Wang, L. Tong, L. Yang, Z. Wang, K. Qi, S. Deng, Q. Li, X. Bai, F. Ding and J. Zhang, Nature, 2017, 543, 234-238.

11 B. Yu, C. Liu, P. X. Hou, Y. Tian, S. Li, B. Liu, F. Li, E. I. Kauppinen and H. M. Cheng, J. Am. Chem. Soc., 2011, 133, 5232-5235.

12 B. Liu, H. Jiang, A. V. Krasheninnikov, A. G. Nasibulin, W. Ren, C. Liu, E. I. Kauppinen and H. M. Cheng, Small, 2013, 9, 1379-1386.

13 Z. Zhu, H. Jiang, T. Susi, A. G. Nasibulin and E. I. Kauppinen, J. Am. Chem. Soc., 2011, 133, 1224-1227.

14 Y. Liao, H. Jiang, N. Wei, P. Laiho, Q. Zhang, S. A. Khan and E. I. Kauppinen, J. Am. Chem. Soc., 2018, 140, 97979800.

15 B. Liu, H. Jiang, A. V. Krasheninnikov, A. G. Nasibulin, W. Ren, C. Liu, E. I. Kauppinen and H. M. Cheng, Small, 2013, 9, 1379-1386.

16 B. Liu, F. Wu, H. Gui, M. Zheng and C. Zhou, ACS Nano, 2017, 11, 31-53.

17 X. Wang, M. He and F. Ding, Mater. Today, 2018, 21, 845-860.
18 A. Kaskela, A. G. Nasibulin, M. Y. Timmermans, B. Aitchison, A. Papadimitratos, Y. Tian, Z. Zhu, H. Jiang, D. P. Brown, A. Zakhidov and E. I. Kauppinen, Nano Lett., 2010, 10, 4349-4355.

19 K. Hata, D. N. Futaba, K. Mizuno, T. Namai, M. Yumura and S. Iijima, Science, 2004, 306, 1362-1364.

20 A. R. Harutyunyan, G. Chen, T. M. Paronyan, E. M. Pigos, O. A. Kuznetsov, K. Hewaparakrama, S. M. Kim, D. Zakharov, E. A. Stach and G. U. Sumanasekera, Science, 2009, 326, 116-120.

21 F. Yang, X. Wang, J. Si, X. Zhao, K. Qi, C. Jin, Z. Zhang, M. Li, D. Zhang, J. Yang, Z. Zhang, Z. Xu, L. M. Peng, X. Bai and Y. Li, ACS Nano, 2017, 11, 186-193.

22 A. Hussain, Y. Liao, Q. Zhang, E. X. Ding, P. Laiho, S. Ahmad, N. Wei, Y. Tian, H. Jiang and E. I. Kauppinen, Nanoscale, 2018, 10, 9752-9759.

23 A. Kaskela, Doctoral Thesis, Aalto University, 2013.

24 A. Moisala, A. G. Nasibulin, S. D. Shandakov, H. Jiang and E. I. Kauppinen, Carbon, 2005, 43, 2066-2074.

25 G. A. Rance, D. H. Marsh, R. J. Nicholas and A. N. Khlobystov, Chem. Phys. Lett., 2010, 493, 19-23.

26 Y. Tian, H. Jiang, J. V. Pfaler, Z. Zhu, A. G. Nasibulin, T. Nikitin, B. Aitchison, L. Khriachtchev, D. P. Brown and E. I. Kauppinen, J. Phys. Chem. Lett., 2010, 1, 1143-1148.

27 H. Jiang, A. G. Nasibulin, D. P. Brown and E. I. Kauppinen, Carbon, 2007, 45, 662-667.

28 F. Ding, A. R. Harutyunyan and B. I. Yakobson, Proc. Natl. Acad. Sci. U. S. A., 2009, 106, 2506-2509.

29 Y. Tian, M. Y. Timmermans, S. Kivistö, A. G. Nasibulin, Z. Zhu, H. Jiang, O. G. Okhotnikov and E. I. Kauppinen, Nano Res., 2011, 4, 807-815.

30 W. Zhou, S. Zhan, L. Ding and J. Liu, J. Am. Chem. Soc., 2012, 134, 14019-14026.

31 R. Kimura, Y. Hijikata, C. A. Eveleens, A. J. Page and S. Irle, J. Comput. Chem., 2019, 40, 375-380.

32 M. F. C. Fiawoo, A. M. Bonnot, H. Amara, C. Bichara, J. Thibault-Pénisson and A. Loiseau, Phys. Rev. Lett., 2012, 108, 195503.

33 W. C. Swope, H. C. Andersen, P. H. Berens and K. R. Wilson, J. Chem. Phys., 1995, 32, 505.

34 S. Nosé, J. Chem. Phys., 1984, 81, 511-519.

35 W. G. Hoover, Phys. Rev. A, 1985, 31, 1695-1697.

36 M. Elstner, D. Porezag, G. Jungnickel, J. Elsner, M. Haugk and T. Frauenheim, Phys. Rev. B: Condens. Matter Mater. Phys., 1998, 58, 7260-7268.

37 G. Zheng, H. A. Witek, P. Bobadova-Parvanova, S. Irle, D. G. Musaev, R. Prabhakar, K. Morokuma, M. Lundberg, M. Elstner, C. Köhler and T. Frauenheim, J. Chem. Theory Comput., 2007, 3, 1349-1367.

38 M. Weinert and J. W. Davenport, Phys. Rev. B: Condens. Matter Mater. Phys., 1992, 45, 13709.

39 R. M. Wentzcovitch, J. L. Martins and P. B. Allen, Phys. Rev. B: Condens. Matter Mater. Phys., 1998, 45, 11372.

40 F. Wagner, T. Laloyaux and M. Scheffler, Phys. Rev. B: Condens. Matter Mater. Phys., 1998, 57, 2102. 